\title{
Análise de vibração em derriçadora de café portátil
}

Analysis of vibration in a coffee harvester portable

\section{Resumo}

A vibração é uma das variáveis mais importantes a serem entendidas e interpretadas no projeto e meIhoria de máquinas. As derriçadoras portáteis têm se mostrado de suma importância na colheita do café para o pequeno e médio produtor. A vibração inerente do funcionamento das ferramentas elétricas manuais se propaga pelas mãos e braços do operador, gerando preocupações quanto a saúde ocupacional dos trabalhadores expostos a essa variável ergonômica. $\bigcirc$ presente estudo teve como objetivo avaliar os níveis de vibração transmitidos por derriçadoras de café manual, baseando-se no comportamento (aceleração) da empunhadura em função de cada tipo de ponteira utilizada juntamente com a variação das rotações de trabalho. Os dados experimentais obtidos foram analisados através do software g4 e manipulados estatisticamente via planilha eletrônica e confrontados com a norma vigente. Os resultados mostraram que os níveis de aceleração na condição de alta rotação, extrapolam o que é permitido pela norma para uma jornada de 8 horas de trabalho, no entanto na condição de baixa rotação os níveis de vibração do equipamento atende o que é preconizado pelas normas.

\section{Abstract}

Vibration is one of the most important variables to be understood and interpreted in the design and improvement of machines. Portable coffee harvester have proved to be of utmost importance in the coffee harvest for small and medium-sized producers. The inherent vibration of the handheld power tools propagates through the hands and arms of the operator, generating concerns about the occupational health of workers exposed to this ergonomic variable. The present study aimed to evaluate the vibration levels transmitted by manual coffee harvester, based on the behavior (acceleration) of the handle as a function of each type of nozzle used together with the variation of the working rotations. The experimental data obtained were analyzed using the 94 software and manipulated statistically via spreadsheet and compared to the current standard. The results showed that the acceleration levels in the high rotation condition exceed what is allowed by the standard for an 8-hour working day, however in the low rotation condition the vibration levels of the equipment meet the standards recommended by the standards. 


\section{INTRODUÇÃO}

Segundo Campanhia Nacional de Abastecimento, CONAB (2017), o Brasil tem cerca de 2,21 milhões de hectares de área plantada com café, e esse número vem crescendo com o passar dos anos devido, principalmente ao uso de novas tecnologias nessa cultura. Minas Gerais é o estado brasileiro que mais produz, seguido de Espirito Santo, São Paulo e Bahia. Juntos estes somam cerca de 90\% da produção brasileira, que é estimada em $24,02 \mathrm{sc} /$ ha.

Atualmente, $80 \%$ do café produzido no mundo é oriundo da cafeicultura de montanha. No Brasil esse tipo de cultivo representa cerca de $40 \%$ da população cafeeira, apesar do país apresentar a maior área mecanizada do mundo, a cafeicultura de montanha abrange 1,5 bilhões de cafeeiro (DE SOUZA, 2006; ROMERO, 2013). Matiello et al. (2005) citam que grande parte das áreas cafeeiras ainda são colhidas manualmente, principalmente quando se trata de regiões com declividades superiores a $20 \%$.

Por se tratar de uma cultura perene, como a seringueira, cacau e o dendê, a cafeicultura demanda muita mão de obra no direcionamento da lavoura, cerca de $35 \%$ a $50 \%$ dos custos da saca de café são atribuidos sem dúvidas a colheita (ROMERO, 2013). Para Oliveira et al. (2007) a colheita é uma das etapas mais onerosa e complexa e está fortemente associada a qualidade do produto.

A mecanização na agricultura é um vies para os pequenos e médios produtores se manterem na atividade e atender a demanda, visto que, a derriça manual pode representar cerca de $75 \%$ do tempo necessário para a colheita, o que de fato gera um alto custo (INAMASU; ANDRADE, 1997; SALVADOR et al., 1998; SILVA et al., 1997).

Embora os mecanismo causadores de distúrbios não estejam claros, justifica-se a avaliação dos níveis de vibração em derriçadoras, como forma de embasamento para o desenvolvimento de mecanismo que visam isolar o operador dos efeitos gerados pela vibração.

Com base nesse contexto, o presente trabalho tem como objetivo avaliar os níveis de vibração transmitidos por derriçadoras de café manual, baseando-se no comportamento (aceleração) da empunhadura em função de cada tipo de ponteira utilizada juntamente com a variação das rotações, através da coleta e análise dos sinais de aceleração.

\section{REFERENCIAL TEÓRICO}

Baseadas no método de vibração mecânica, ou seja, transferência de energia mecânica, as primeiras colhedoras de café surgiram na década de oitenta, no entanto estudos referentes a colheita mecanizada de produtos agricolas tiveram origem em meados de setenta (FAYA et al., 1979).

A colheita mecanizada de frutos de café é realizada de forma eficiente a partir da associação de fatores como frequência e amplitude de vibração, dessa forma pode-se transferir energia vibracio- 
nal para o sistema fruto-pedúnculo a qual seja suficiente para o desprendimento dos frutos. Conhecendo as propriedades dinâmicas sob excitação por vibração do sistema fruto-pedúnculo, podem-se combinar níveis de Desenvolvimento de uma derriçadora portátil para colheita de café frequência e amplitude adequados para a realização da colheita seletiva ou total dos frutos (SANTOS, 2010; SRIVASTAVA et al., 1996; CIRO, 2001).

Garcia e Fioravante (2002) realizaram um ensaio de colheita mecanizada e no ano seguinte através das análises das produções notaram que não houve prejuízos na safra. Barbosa et al. (2005) num estudo de desempenho em derriçadoras portáteis, concluíram que a derriça mecanizada apresentou níveis de desempenho superiores a derriça manual, atribuindo ainda os melhores resultados a equipamentos com frequência superior a $50 \mathrm{~Hz}$, e lavouras com densidade de ramos menores.

Níveis de desempenho maiores para a colheita seletivas são verificados quando se empregam duas máquinas simultaneamente, em uma mesma linha de plantio, afirmam De Souza et al. (2006).

Diferentes são os tipos de acionamento das derriçadoras portáteis, a fonte de potência pode ser pneumática, elétrica ou por combustão interna, sendo que acima de $735 \mathrm{~W}$ para os equipamentos pneumáticos e por combustão interna e de $500 \mathrm{~W}$ para os motores elétricos (PORTO, 1996; CARVALHO et al., 2000; BARBOSA et al., 2005).

Atrelado ao desenvolvimento de tecnologia para o processo de colheita, estão os números de acidentes e doenças ocupacionais, o que gera preocupação com os trabalhadores expostos, dessa forma é de fundamental importância conhecer os níveis de ruído e vibração gerados em maquinários agrícolas para que se possam gerar soluções que visam isolar o trabalhador dos efeitos dessas variáveis ergonômicas. Quando a máquina proporciona conforto ao operador, seu desempenho aumenta sensivelmente, portanto é de fundamental importância conhecer os aspectos de funcionamento e operação.

A ergonomia estuda a adaptação da máquina ao homem e o seu principal objetivo é modificar os sistemas de trabalho para adequar à atividade nele existente, ou seja, auxiliar em projeto de máquinas de maneira a acomodar a variabilidade natural, associando-a ao desempenho humano, atingindo o propósito geral do sistema com segurança e produtividade (HASSALL, M. et al., 2015). A preocupação dos fabricantes com esse estudo é muito recente, visto que os consumidores começaram a exigir melhorias ergonômicas em decorrência de alterações fisiológicas e psicológicas que comprometem a saúde do trabalhador e a sua eficiência no trabalho (SCHLOSSER, 2002).

Por se tratar de uma variável que causa desconforto quase que instantâneo, o ruído é um dos itens mais avaliados em trabalhos relacionados à ergonomia. Em derriçadoras manuais esses níveis extrapolam o que é estabelecido pela Norma NB 95 (NBR 10152) e pela NR-15 (CLT), sendo 85 dBA para uma exposição de $8 \mathrm{~h}$ diária.

Cunha et al. (2002) e Souza et al. (2005) avaliaram os níveis de ruído emitido por três derriçadoras portáteis, concluindo que as mesmas apresentaram níveis de ruído acima dos limites estabelecidos. 
Já a variável vibração é tratada apenas do ponto de vista de rendimento do equipamento, porém quando a vibração é transmitida ao corpo humano, pode acarretar em doenças ocupacionais, as quais envolvem o sistema vascular, neurológico e musculoesquelético. Os efeitos sobre o corpo humano dependem, principalmente, da frequência, magnitude, direção, área de contato e tempo de exposição (THOMPSON et al., 2011; BAAD; QAIMI, 2016 );

A faixa de frequências na qual o corpo humano apresenta maior sensibilidade é de 1 a $80 \mathrm{~Hz}$, a exposição excessiva a vibrações pode levar a lesões arteriais ou nervosas em operadores. Isso pode provocar a doença dos dedos brancos (fenômeno de Raynaud), cujo sintomas incluem sensações de formigamento e queimadura seguido de perda de cor e dormência nos dedos (GERGES, 2000; XIMENES, 2006) .

\section{PROCEDIMENTOS METODOLÓGICOS}

A metodologia utilizada no presente estudo foi composta de experimentação em campo com levantamento de dados sobre os fenômenos estudados, onde aplicou-se técnicas estatísticas que deram o embasamento para chegar nos resultados, os quais foram comparados com a legislação vigente. Para o tratamento dos dados fez-se o uso de planilhas eletrônicas e o software G4.

Para realização dos ensaios, utilizou-se uma derriçadora monoclindro (Figura 1) com 1,2 cv de potência, $23 \mathrm{cc}$ e reservatório com capacidade para 0,6 litros. O equipamento é composto por uma fonte de potência, uma haste tubular em alumínio, onde é acoplado a haste foram utilizadas 2 ponteiras distintas (Figura 2), sendo elas Ponteira A (12 dedos) e Ponteira B (14 dedos). O princípio de funcionamento das ponteiras são idênticos, e apresentam uma massa de aproximadamente 1,8 kg.

Para medir a aceleração transmitida ao sistema mãos e braços, utilizou-se um acelerômetro triaxial, modelo SEN040F acoplado ao sistema de aquisição HVM200, por meio de um cabo, cujo fabricante é a Larson Davis.

Como forma de manter os mesmos parâmetros durante a realização de todos os ensaios, a rotação da derriçadora foi controlada por meio de um tacômetro digital, modelo PET304 fabricado pela empresa ECHO. O aparelho foi fixado ao cabo de vela do motor através de um mordente, assim foi possível fazer a leitura da rotação ao longo do tempo, de forma a controlar aceleração do motor e manter uma faixa de rotação para todos os ensaios.

Um operador qualificado na categoria manuseou a derriçadora, cujo acelerômetro, juntamente com o adaptador foram fixados na empunhadura inferior conforme a norma NHO 10 (2013), respeitando a localização dos eixos, conforme é mostrado na Figura 1. Todas as medidas de proteção foram implementadas, de forma a manter a integridade física do operador. O projeto foi aprovado pelo Comitê de Ética em Pesquisa da Faculdade de Arquitetura e Artes da Unesp, Campus Bauru, 
CAAE: 87454818.4.000.5663. Para a aquisição dos sinais durante os ensaios, foram consideradas as condições em que a derriçadora encontrava-se em funcionamento, porém não estava em trabalho de derriça.

Figura 1 - Derriçadora portátil

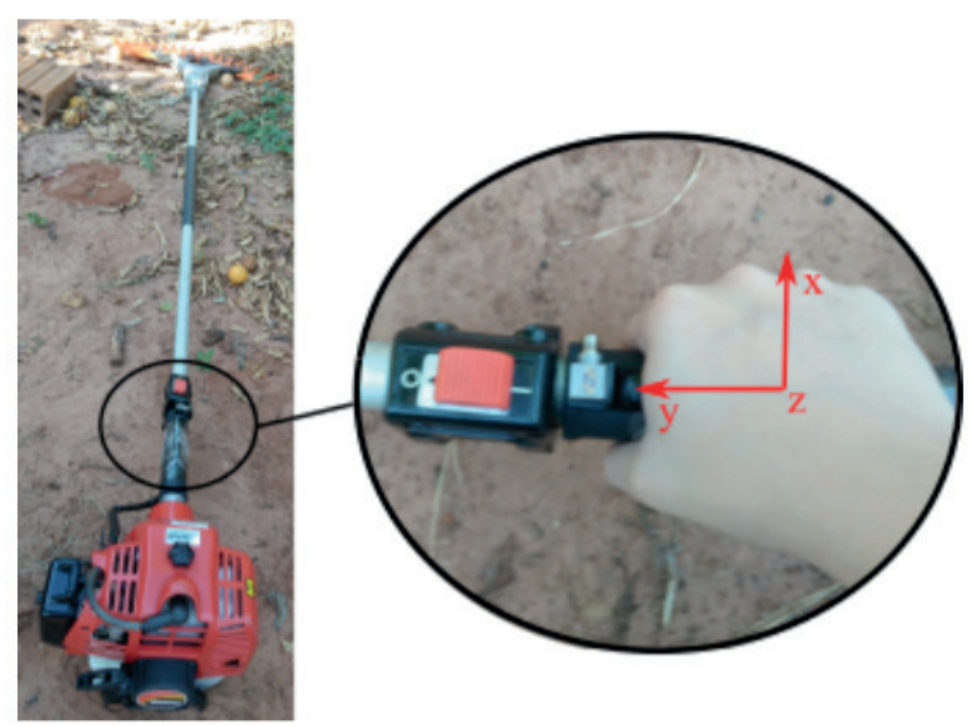

Fonte: Os autores.

Figura 2 - Ponteiras

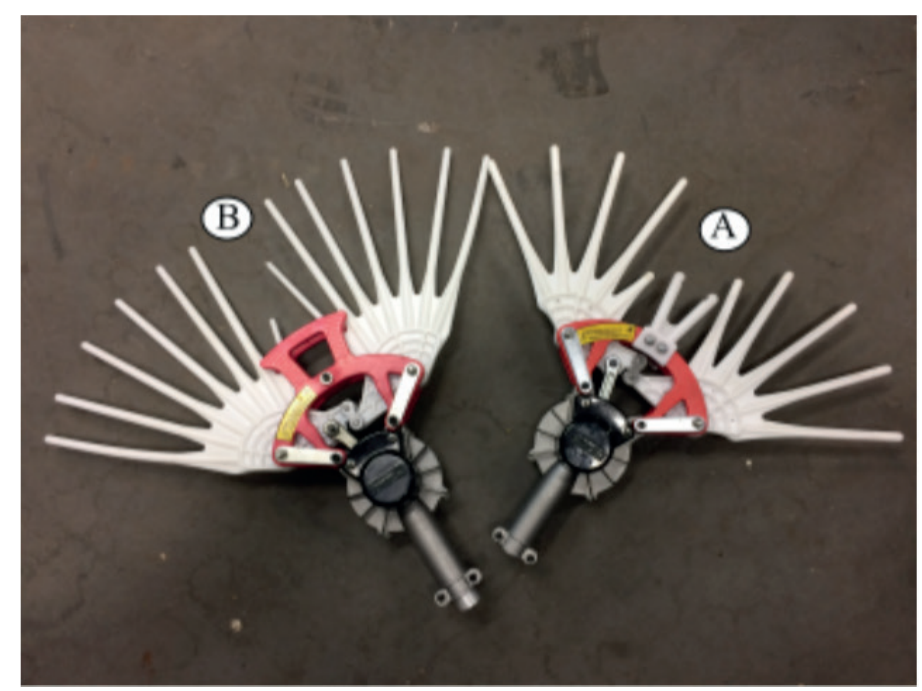

Fonte: Os autores.

Foram realizados 4 ensaios, sendo 2 em baixa rotação e 2 em alta rotação, ambos com as ponteiras A e B (Figura 2). Para cada ensaio foram feitas 3 repetições com duração de 1 minuto por repetição, portanto a frequência de aquisição de dados foi determinada em $60 \mathrm{~Hz}$, ou seja, o módulo captava uma leitura do sensor (aceleração em r.m.s.) a cada segundo. Todos os dados foram armazenados e 
tratados estatisticamente a fim de extrair informações relevantes do comportamento da aceleração do equipamento. A Tabela 1 apresenta a sequência de ensaios, bem como a configuração do mesmo.

Tabela 1 - Sequência dos ensaios.

\begin{tabular}{ccc|cc}
\hline \multicolumn{3}{c|}{ Ponteira } & \multicolumn{2}{c}{ Rotação } \\
\hline Ensaio & A & B & Baixa & Alta \\
& & & & \\
\hline $\mathbf{1}^{\circ}$ & $\mathrm{X}$ & & $\mathrm{X}$ & \\
\hline $\mathbf{2}^{\circ}$ & $\mathrm{X}$ & & & $\mathrm{X}$ \\
& & & & \\
\hline $\mathbf{3}^{\circ}$ & & $\mathrm{X}$ & $\mathrm{X}$ & \\
\hline $\mathbf{4}^{\circ}$ & & $\mathrm{X}$ & & $\mathrm{X}$ \\
& & & & \\
\hline
\end{tabular}

Fonte: Os autores.

Definiu-se como baixa rotação o ponto do motor o qual as ponteiras não sofressem deslocamento, porém estavam na iminência do movimento. Já a alta rotação foi estipulada de acordo com o manual do fabricante, sendo ela cerca de $11000 \mathrm{rpm}$ em plena carga.

Um método de representação gráfica, Boxplot, foi utilizado, onde o mesmo fornece informações sobre o conjunto de dados, tais como localização, dispersão, assimetria, comprimento da cauda e medidas discrepantes (outliers). Após o tratamento estatístico, os dados foram interpretados tomando como base a norma vigente no país, ou seja, NHO 10 a qual faz referência a norma internacional ISO 5349:2001 parte 1 e 2 e a Diretiva Europeia 2002/44/EC que estipula o nível de ação e limite de exposição da vibração.

Avaliou-se o tempo que o trabalhador pode operar o equipamento sem que haja prejuízo à saúde (nível de ação), bem como, o tempo máximo de exposição, ou seja, o tempo máximo até que atinja o limite de exposição proposto pela Diretiva Europeia, conforme mostrado na Tabela 2.

Tabela 2 - Critério de julgamento e tomada de decisão

\begin{tabular}{ccc} 
& Nivel de ação $\left(\mathrm{n}: \mathrm{s} / \mathrm{s}^{2}\right)$ & Linite de Exposição $\left(\mathrm{r}: / \mathrm{s}^{2}\right)$ \\
\hline Mãos e Braços & 2,5 & 5,0 \\
& & \\
\hline
\end{tabular}

Fonte: Diretiva Europeia 2002/44/EC (2002).

Calculou-se o tempo que o operador pode ficar exposto às vibrações obtidas durante os testes, para uma jornada de trabalho de 8 horas diárias. Para isso, utilizou-se a Equação (1) e os níveis estabelecidos 
pela Diretiva Europeia 2002/44/EC (2002) para que não haja prejuízo à saúde do operador.

$$
A(8)=A_{e q} \sqrt{\frac{\mathrm{T}}{\mathrm{T}_{0}}}
$$

Onde:

$\mathrm{A}(8)$ - projeção de dose para 8 horas;

$A_{\text {eq }}$ - valor de aceleração da vibração transmitida à mão (raiz média quadrática das componentes $\mathrm{x}$, y e $\mathrm{z})$, em metros por segundo ao quadrado $\left(\mathrm{m} / \mathrm{s}^{2}\right)$ e $\mathrm{rms}$;

$\mathrm{T}$ - duração total para a exposição da vibração $\mathrm{A}_{\text {eq }}$;

To - duração referente a 8 horas (28800s).

\section{RESULTADOS E DISCUSSÕES}

Os resultados dos ensaios com a derriçadeira estão apresentados a seguir, onde é possível notar na Figura 3, a qual faz referência a uma análise de dispersão dos dados, que os ensaios 1 e 3 apresentaram uma baixa dispersão. Já os ensaios 2 e 4 apresentaram uma maior dispersão entre os dados, comportamento esse atribuído ao fato do equipamento estar sendo operado manualmente e depender da precisão do operar em travar o gatilho de forma constante, fato que não ocorre com os ensaios em baixa rotação, pois o operador apenas segura o equipamento, uma vez que o mesmo já está regulado de forma constante.

Figura 3 - Comportamento da aceleração resultante.

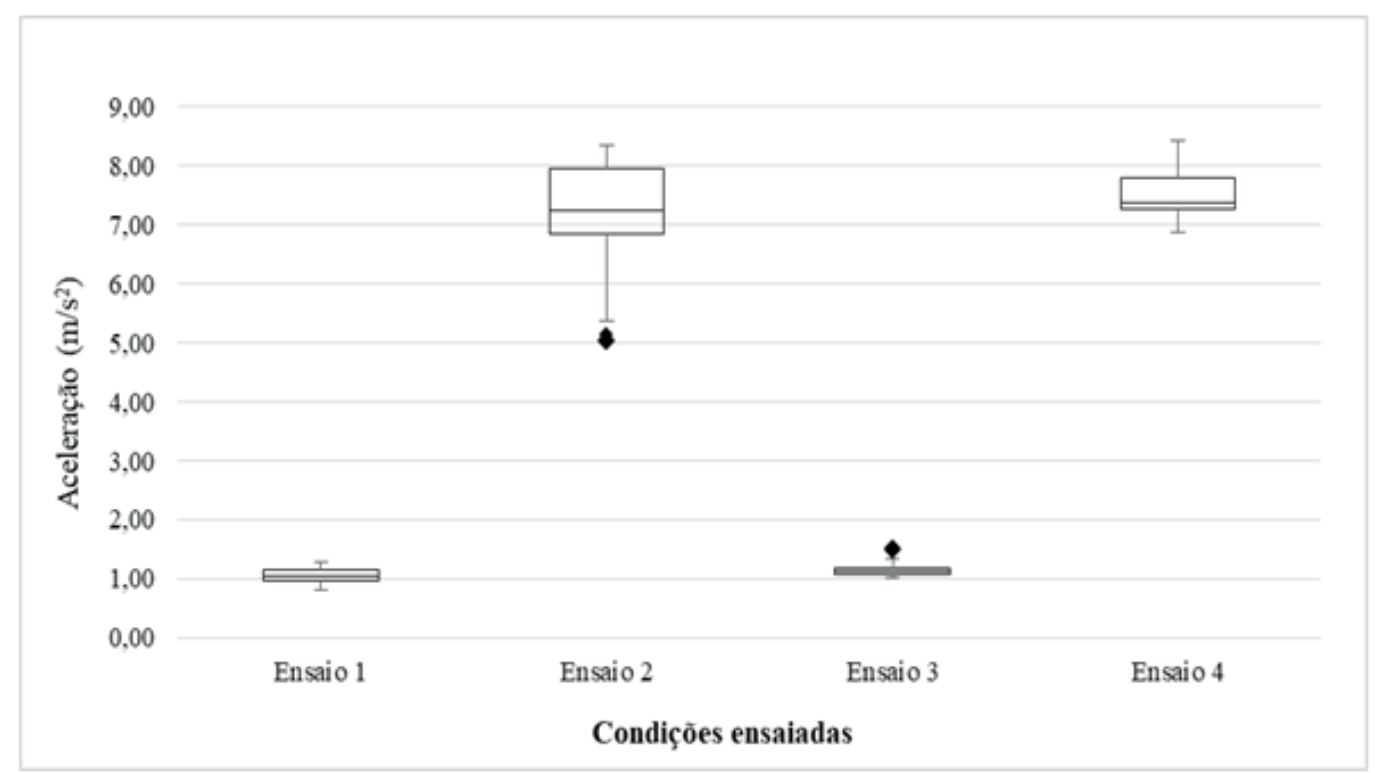

Fonte: Os autores. 
No gráfico apresentado a seguir pela Figura 4, nota-se com mais clareza a variabilidade da aceleração instantânea, causada pelo manuseio do gatilho nos ensaios 2 e 4, onde também vizualiza-se os outiliers, ou seja, os pontos que não apresentam um comportamento padrão, mas que fazem parte dos ensaios de campo. Os ensaios 1 e 3 apresentam um comportamento linear e constante mostrando que a condições ensaiadas tiveram seus parâmetros bem controlados.

Figura 4 - Comportamento da aceleração instantânea.

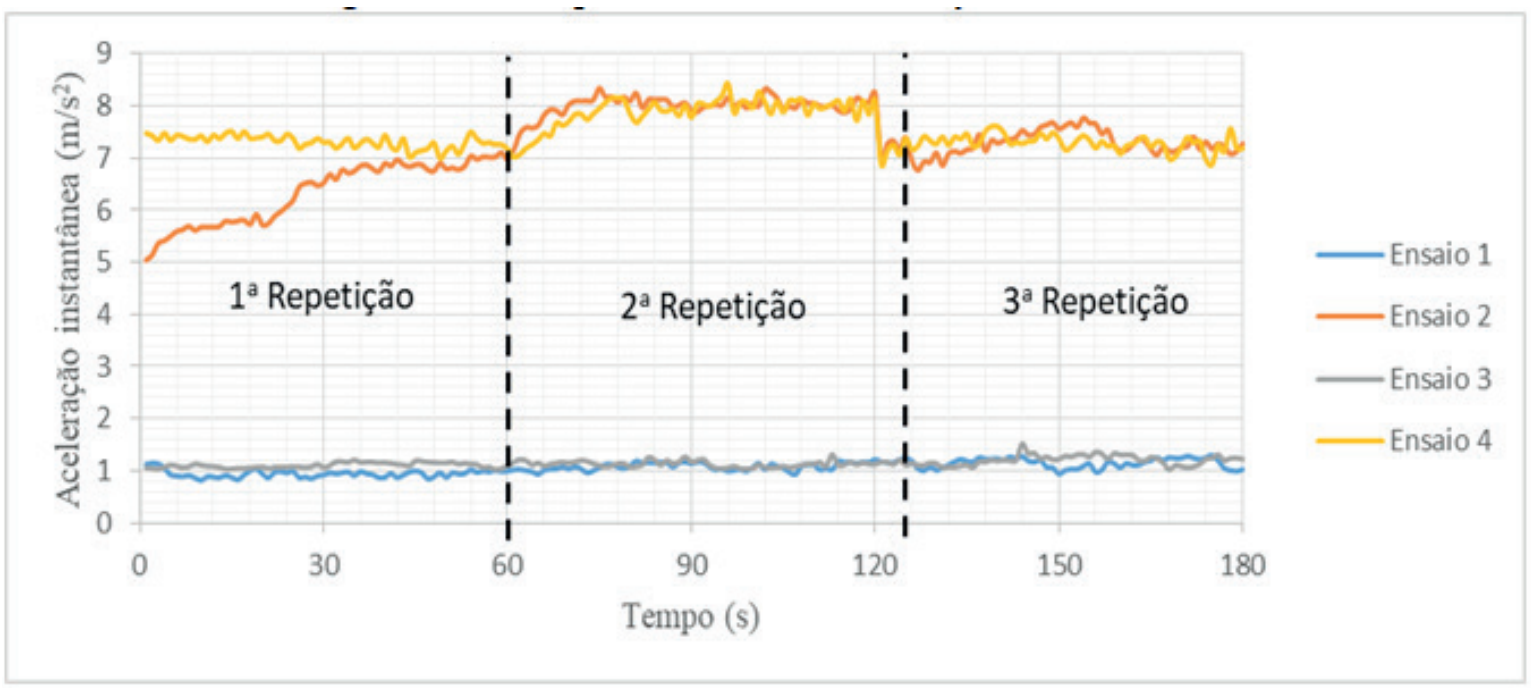

Fonte: Os autores.

Para os ensaios apresentados, obteve-se níveis de rotações médias que variaram entre 3053,33 rpm a 10155,56 rpm, sendo esses valores atribuidos a baixa e alta rotação respectivamente. Ambos os comportamentos apresentados, mostram os extremos da operação de derriça, ou seja, são situações nas quais o operador está submetido de forma intermitente, mas que se somadas durante a jornada de trabalho tem efeitos consideráveis.

É possivel notar a diferença entre as acelerações médias dos ensaios de baixa e alta rotação, parte dessa diferença, pode ser atribuida as variações entre os fatores geométrico das ponteira, tais como massa e geometria dos componentes. A Figura 5 apresenta essa diferença, que chega a ser na ordem de $700 \%$ maiores.

Nota-se também que, nas condições de baixa rotação obteve-se menores magnitudes de aceleração (Ensaio 1 e 3), enquanto que, em alta rotação essa aceleração aumentou, o que era esperado, pois em alta rotação as hastes estão sofrendo oscilação máxima de amplitude.

Foram feitas 3 repetições para cada teste, portanto utilizou-se a média aritmética das repetições para obter a aceleração média para cada teste realizado e, posteriormente, analisar e obter o valor de A eq, mostrado na Figura 5. 
Figura 5 - Aceleração média dos ensaios.

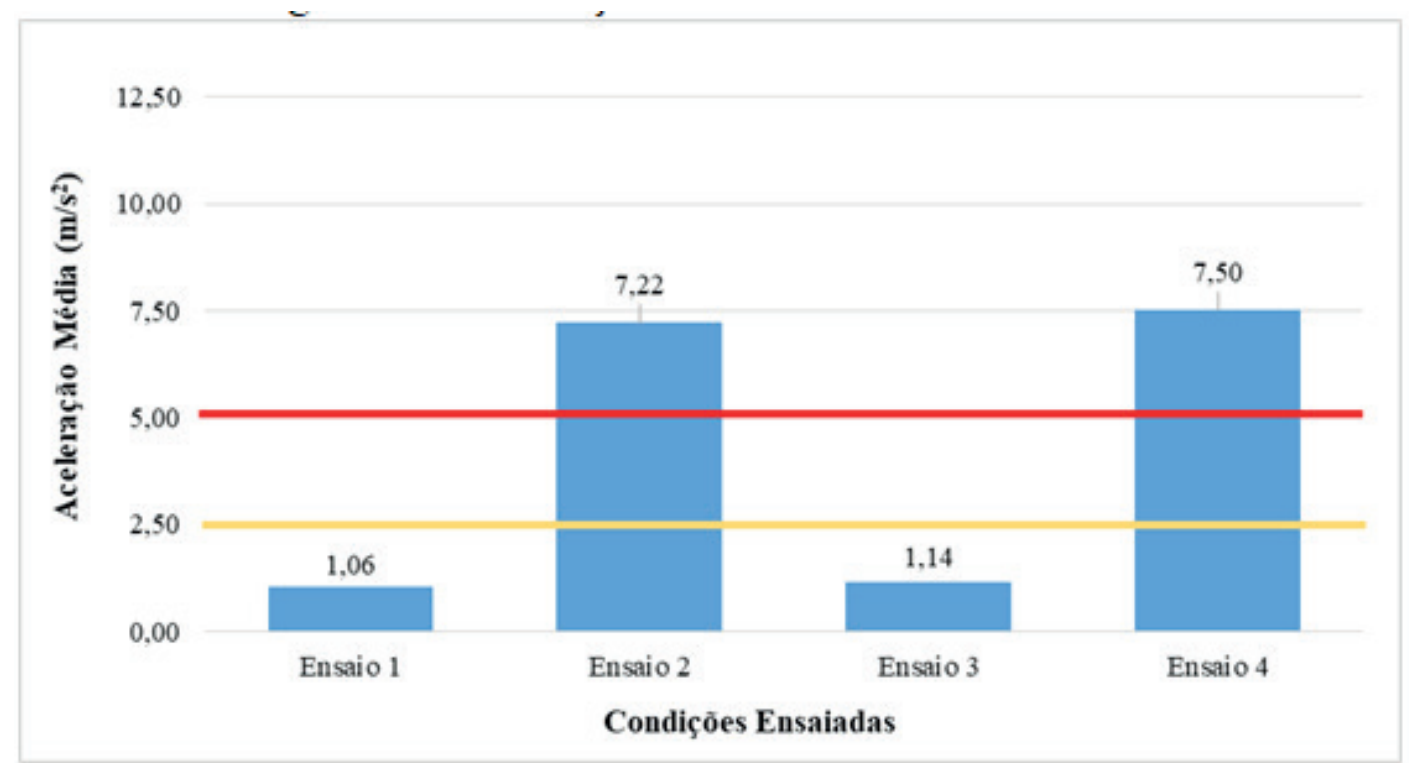

Fonte: Os autores.

Ainda, nota-se que os níveis para os ensaios em baixa rotação (Ensaio 1 e 3) estão de acordo com o nível apresentado na Tabela 2 como aceitáveis (nível de ação), não trazendo malefícios à saúde do operador. Já os ensaios 2 e 4 mostraram-se extremamente prejudiciais, estando acima do limite de exposição, o qual exige adoção imediata de medidas corretivas, conforme é representado na Figura 5 pelas linhas amarela (nível de ação) e vermelha (limite de exposição).

Posteriormente calculou-se o tempo em que o operador da derriçadeira pode ficar exposto à vibração sem que haja prejuízo a saúde para uma jornada de trabalho de 8 horas diárias (nível de ação) e, também, o tempo limite de exposição para que a vibração se torne extremamente prejudicial para o trabalhador (limite de exposição), utilizando a Equação (1).

Figura 6 - Análise de tempo de exposição

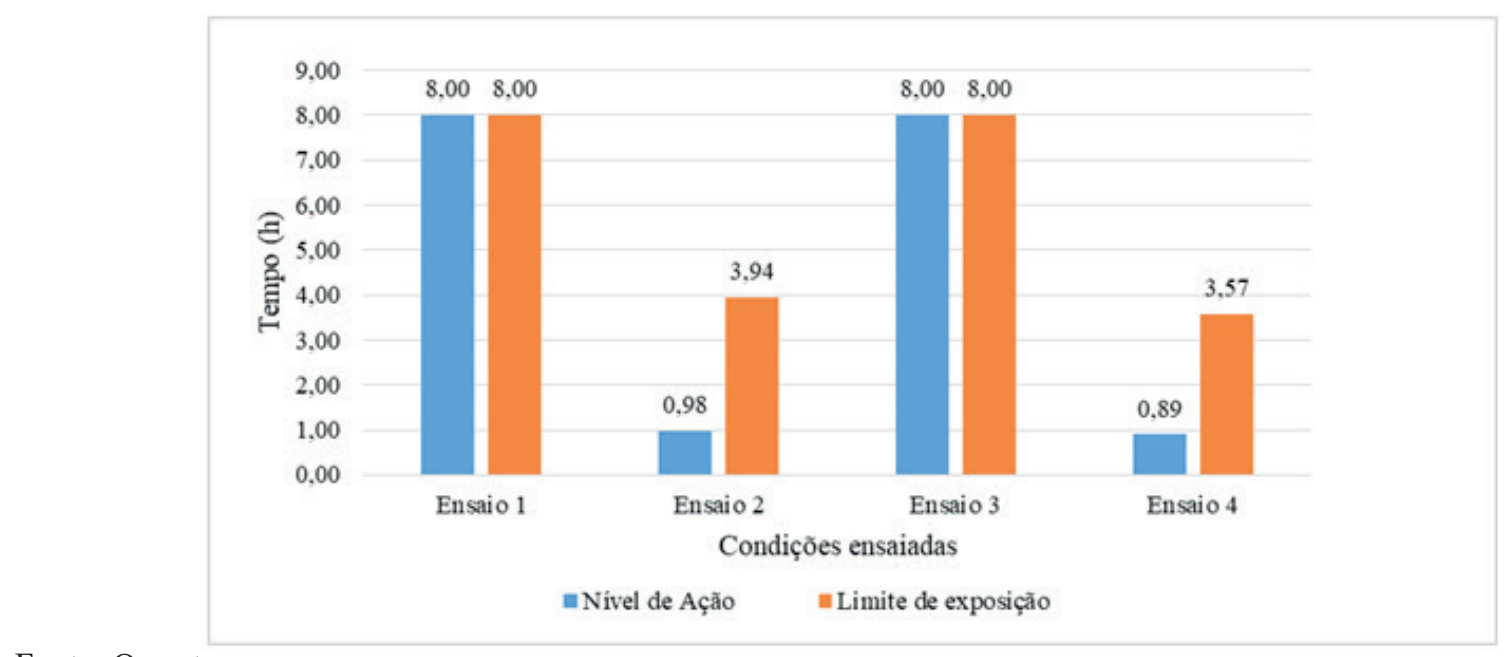

Fonte: Os autores. 
Nota-se que os ensaios 1 e 3 não apresentam riscos à saúde ocupacional do operador, visto que o tempo de exposição atende as 8 horas de uma jornada de trabalho. Já para os ensaios 2 e 4, este valor é muito inferior ao que preconiza a Diretiva Europeia 2002/44/EC tanto para o nível de ação como o limite de exposição, podendo o operador ficar exposto apenas 58 minutos para a condição de operação do ensaio 2 h e 53 minutos para o ensaio 4, quando comparado ao nível de ação e 3 h 56 min e 3 h 34 min para as condições dos ensaios 2 e 4 , respectivamente, quando considerado o limite de exposição.

Após a análise das acelerações de vibração, fez-se os gráficos de espectro de frequência, apresentados na Figura 7, onde é possível notar a concentração da energia de vibração do sistema, e então mapear possíveis causas de vibração excessivas do sistema (RAO, 2009) .

Figura 7 - Espectro de frequência das condições ensaiadas.
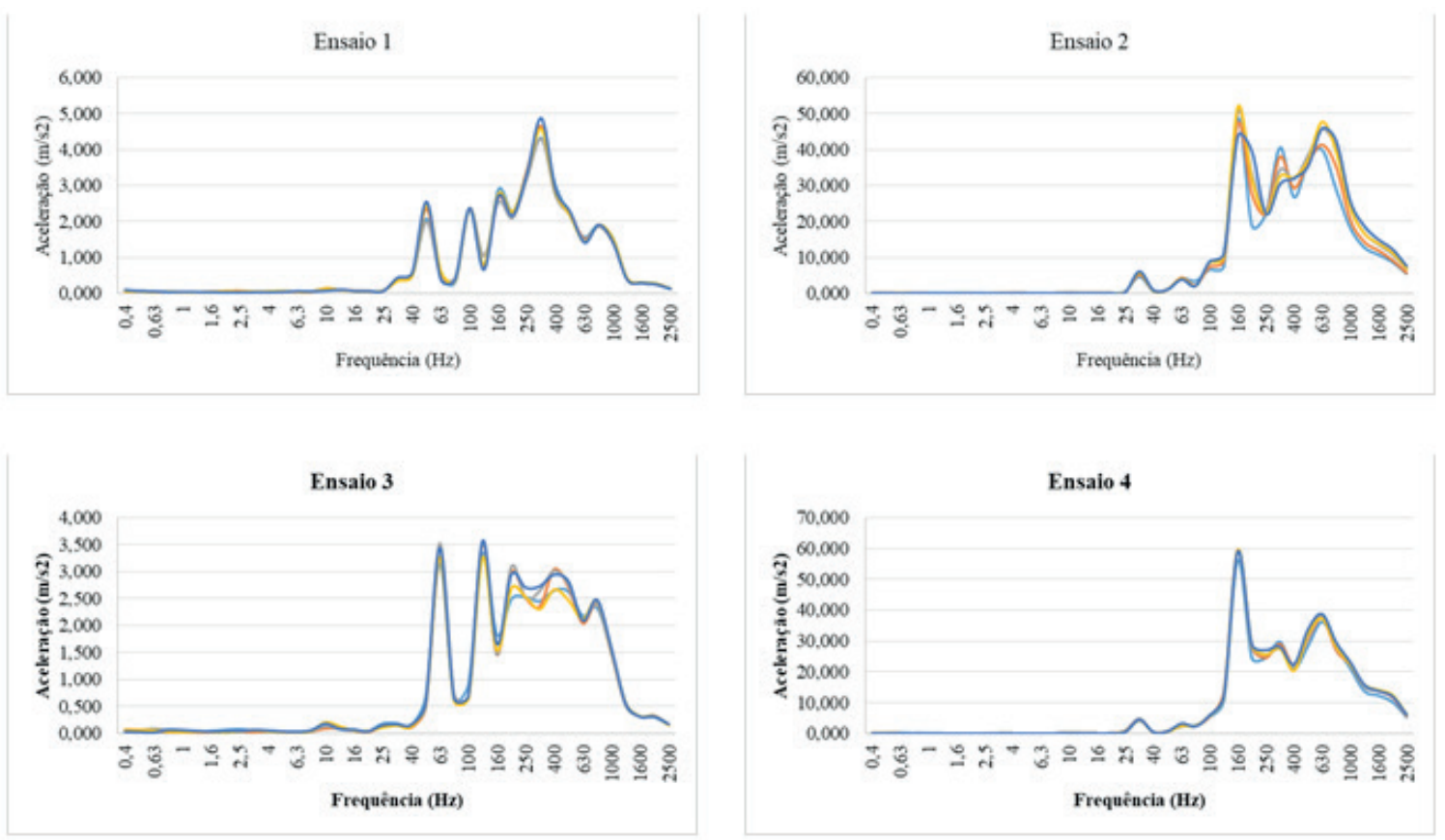

Fonte: Os autores.

Nota-se que cada ensaio obteve-se a concentração de energia em diferentes frequências, sendo que, no ensaio 1, a concentração de energia se dá entre 250 e $400 \mathrm{~Hz}$, enquanto que no ensaio 2 a concentração está ao redor de $160 \mathrm{~Hz}$ e entre 630 e $1000 \mathrm{~Hz}$, para o ensaio 3, entorno de $63 \mathrm{~Hz}$ e 160 $\mathrm{Hz}$ e para o ensaio 4, próximo a $160 \mathrm{~Hz}$. Uma vez que o corpo humano sofre com a sensibilidade na faixa de 1 a $80 \mathrm{~Hz}$ (GERGES, 2000), o ensaio 3 apresenta riscos à saúde ocupacional do operador, visto que o mesmo teve a concentração de energia nesta faixa de frequência. Já para os demais ensaios, o equipamento opera em altas frequências, o que segundo a norma em questão atribui fatores de ponderação menores para este caso, portanto os prejuízos à saúde do operador são menores, quando 
analisado do ponto de vista de frequência.

\section{CONCLUSÕES}

A partir dos resultados encontrados, pode-se concluir que a derriçadora, quando utilizada em baixa rotação, não trará malefícios a saúde ocupacional do operador, porém deve-se atentar a carga horária de trabalho para operações com alta rotação, pois os tempos exposição à vibração, calculados são muito inferiores as 8 horas de uma jornada de trabalho padrão, e desta forma, pode trazer grandes prejuízos a saúde do operador segundo as diretrizes das normas avaliadas. Já para análise de frequência de vibração, os resultados mostraram que em alta rotação a concentração de energia de vibração se dá em altas freqüências as quais tem um fator de ponderação menores, portanto são menos prejudiciais ao operador, entretanto em um dos ensaios, em baixa rotação, obteve-se concentração de energia em uma frequência extremamente prejudicial ao corpo humano, segundo curva de ponderação, devendo atentar-se a essa situação.

Ainda, conclui-se que para este tipo de máquina agrícola, a variável aceleração tem um impacto maior sobre os efeitos da vibração na saúde ocupacional do operador.

Recomenda-se, a alteração do nível de marcha lenta do equipamento, fazendo com que a baixa rotação do mesmo seja superior à utilizada neste trabalho, desta forma a concentração de energia de vibração do equipamento para esta condição seria elevada, saindo da zona de frequências de risco à saúde ocupacional.

Sugere-se a realização de novos ensaios considerando a alteração do nível de marcha lenta, bem como a utilização de ponteiras com valores de massa distintos, uma vez que, essa variável tem influência direta nos níveis de vibração. E ainda, a realização de ensaios utilizando manoplas com materiais que permitam menor transmissibilidade da vibração o sistema mãos e braços do operador.

\section{Referências}

BAAD, S. M.; QAIMI, M. G.; A review on hand arm vibration with special reference to motorcycle handlebar. Indian Jounal of Research, v.5, n.6, p.396-398, 2016

BARBOSA, J. A.; SALVADOR, N.; SILVA, F. M. da. Desempenho operacional de derriçadores mecânicos portáteis, em diferentes condições de lavouras cafeeiras. Revista Brasileira de Engenharia Agrícola e Ambiental, v.9, n.1, p.129-132 2005.

BRASIL. Norma Regulamentadora N. 15. 2018. Disponível em: https://enit.trabalho.gov.br/portal/images/Arquivos_SST/SST_NR/NR-15-Anexo-01.pdf. Acesso em 05 mai 2018. 
CARVALHO, L. T. et al. Desenvolvimento de uma derriçadora portátil para colheita de café. In: SIMPÓSIO DE PESQUISA DOS CAFÉS DO BRASIL. 2000. Disponível em: http://www.sbicafe. ufv.br/bitstream/handle/123456789/644/155537_Art290f.pdf?sequence=1. Acesso em 05 mai 2018.

CIRO, H. J. Coffee harvesting I: determination of the natural frequencies of the fruit stem system in coffee trees. Applied Engineering in Agriculture, v. 17, n. 4, p. 475-479, 2001.

CONAB - Companhia Nacional de Abastecimento. Acompanhamento da safra brasileira. Segunda estimativa Safra Café 2017. Brasília: Ministério da Agricultura, Pecuária e Abastecimento, 2017. 106p. Disponível em: http://www.conab.gov.br/conteudos.php?a=1253\&amp;t=2. Acesso em 05 de agosto de 2017.

CUNHA, J. P. A. R.; FERNANDES, H. C.; BARBOSA, J. A. Avaliação dos níveis de ruído emitidos por derriçadoras portáteis para colheita de café. In: CONGRESSO BRASILEIRO DE ENGENHARIA AGRÍCOLA. 1, 2002. Anais...Salvador: SBEA, Jaboticabal, 2002.

DE SOUZA, C. M.A.; DE QUEIROZ, D. M.; RAFULL, L. Z. L. Derriçadora portátil na colheita total e seletiva de frutos do cafeeiro. Pesquisa Agropecuária Brasileira, v. 41, n. 11, p. 1637-1642, 2006.

FAYA, J. F. M. et al. Eficiência da colheita mecânica nas variedades Mundo Novo e Catuaí em diferentes condições de lavoura. In: 7. CONGRESSO BRASILEIRO DE PESQUISAS CAFEEIRAS. 7. 1979. Anais...Araxá, MG, 1979.

FUNDACENTRO. Procedimento Técnico-Avaliação da exposição ocupacional a vibrações em mãos e braços. 2012. Disponível em: http://www.fundacentro.gov.br/biblioteca/normas-de-higiene-ocupacional/publicacao/detalhe/2013/4/nho-10-procedimento-tecnico-avaliacao-da-exposicao-ocupacional-a-vibracao-em-maos-e. Acesso em 05 mai 2018.

GARCIA, A. W. R.; FIORAVANTE, N. Efeito do uso de derriçadoras de café portáteis na produtividade do cafeeiro no ano seguinte. In: CONGRESSO BRASILEIRO DE PESQUISAS CAFEEIRAS. 2002. Anais...SIBI CAFÉ. http://www.sbicafe.ufv.br/handle/123456789/3372, 2002.

GERGES, S. N. Y. Ruído: Fundamentos e controle. 2. ed. Florianópolis: NR Editora, 2000. 
HASSALL, M. et al., Human factors and ergonomics. International Encyclopedia of the Social and Behavioral Sciences, v.11, p.297-305, 2015.

INAMASU, R. Y.; ANDRADE, JG de. Teste da nova máquina para derriçar café. In: CONGRESSO BRASILEIRO DE PESQUISA CAFEEIRAS. 23. 1997. Anais...SIBI CAFÉ, Manhuaçu, MG. 1997.

NORMA TÉCNICA. ABNT. 10.152: Níveis de Ruído para Conforto Acústico. Rio de Janeiro, 1987.

MATIELLO, J. B. et al. Cultura de café no Brasil: novo manual de recomendações. Ministério da Agricultura, da Pecuária e do Abastecimento, Brasília, DF (Brasil), 2005.

OLIVEIRA, E. de et al. Influência da colheita mecanizada na produção cafeeira. Ciência Rural, v.37, n.5, p.1466-1470, 2007.

PORTO, F. S. A. Desenvolvimento de uma colhedora portátil de café por sucção aerodinâmica. 1996. 98f. Dissertação (Mestrado em Engenharia Mecânica) -Universidade Estadual Paulista "Júlio de Mesquita Filho", UNESP, Bauru, SP, 1996.

ROMERO, J. C. P. Café de montanha perfaz cerca de 40\% da produção brasileira. Visão Agrícola, n.12, p.90-91, 2013.

SALVADOR, N. et al. Estudo comparativo de sistemas de derriça de café. In: CONGRESSO BRASILEIRO DE PESQUISA CAFEEIRAS. 24. 1998. Anais...SIBI CAFÉ, 1998, p. 227-228.

SILVA, F. M. da et al. Desempenho da operação mecanizada de derriça do café. In: CONGRESSO BRASILEIRO DE PESQUISA CAFEEIRAS. 23. 1997. Anais...SIBI CAFÉ, Manhuaçu, MG. 1997, p. 174-176.

SOUZA, C. et al. Desempenho de derriçadora portátil de frutos do cafeeiro. Engenharia Agrícola, v.25, n.3, p.791-800, 2005

SCHLOSSER, J. F. Tratores agrícolas. Núcleo de Ensaios de Máquinas Agrícolas, Centro de Ciências Rurais, Universidade Federal de Santa Maria, 2002. 207p. (Série Técnica - Módulo I). 
SRIVASTAVA, A. K. et al. Engineering principles of agricultural machines. St. Joseph, Michigan: American society of agricultural engineers, 1993.

THOMPSON, A. et al. Compensation of hand-arm vibration syndrome in Canada. Canadian Acoustics, v. 39, n. 2, p. 112-113, 2011.

XIMENES, G. M. Gestão Ocupacional da vibração no corpo humano, aspectos técnicos e legais relacionados à saúde e segurança. 118f. 2006. Dissertação (Mestrado em Sistemas de Gestão) - Universidade Federal Fluminense, Niterói, RJ, 2006. 